\title{
Cosmology with primordial black holes motivated from extra-dimensional theories
}

\author{
A. S. Majumdar* \\ S. N. Bose National Center for Basic Sciences, Kolkata, India \\ E-mail: archan@bose.res.in
}

We discuss the possibility of the survival of primordial black holes as dark matter candidates in various alternate gravity theories motivated from extra-dimensional scenarios. We show that in particular, braneworld black holes, as well as black holes in scalar-tensor models can survive up to late times by efficient accretion of radiation in the early universe. Constraints on the initial mass spectrum of primordial black holes could be obtained through various cosmological observations.

Black Holes in General Relativity and String Theory

24âĂS 30 August, 2008

Veli LoÅainj, Croatia

${ }^{*}$ Speaker. 


\section{Introduction}

Cosmology with primordial black holes is an interesting subject which is yet to be investigated in full details for different models of black hole formation and cosmological evolution. Black holes could be formed in the early universe through a variety of mechanisms[1]. A widely studied mechanism is that of the cosmological density perturbations generated through inflation[2]. Inflation leads to a scale invariant spectrum of primordial density perturbations which tightly constrains the production rate and the initial mass spectrum of primordial black holes formed through the collapse of density perturbations[3]. Due to the fact that black holes can accrete their surrounding energy as well as radiate energy through the process of Hawking evaporation, the interest in the study of the evolution of primordial black holes[4] ranges over a very big time span starting from their formation and running right through their lifetimes which, in certain cases could even exceed the present age of the universe. A key question concerning primordial black holes is their longevity which depends crucially on the effectiveness of various accretion processes.

Primordial black holes have been studied extensively in the standard cosmological model, though their remain several gaps in our understanding of their formation, evolution and implications. Among the notable cosmolological consequences of surviving primordial black holes, to name a few one could mention the possibility of generation of the baryon asymmetry in the universe[5], and their viability as candidates of cold dark matter[6, 7]. Primordial black holes could also have interesting astrophysical consequences, such as the seeding of supermassive black holes[8].

Recent resurgence of interest in the physics of primordial black holes comes also from the advent of braneworld models of the universe. Indeed, the entire scenario of cosmology with primordial black holes gets modified in the context of braneworld gravity. The modified rate of expansion of the Hubble volume makes it feasible for accretion by the primordial black holes of the surrounding radiation to take place. This feature is primarily responsible for the mass growth and prolonged survival $[9,10]$ of the braneworld black holes. The standard cosmological expansion of the universe is recovered at later times, in order for the observationally established processes such as nucleosynthesis to work out. Various cosmological observations impose constraints on the initial mass spectrum of primordial black holes in the braneworld model[11]. The survival of primordial black holes due to efficient early accretion of radiation could be a feature of other cosmological models too that are motivated from extra-dimensional theories. In this article we will further discuss one such scenario, viz., the evolution of primordial black holes in a scalar-tensor cosmological model[12].

\section{Cosmological evolution of primordial black holes}

We bigin with a brief description of cosmological evolution of primordial black holes. Consider a population of primordial black holes in the early radiation dominated era of the universe. These black holes could be formed by various mechanisms[1] operative in the post-inflationary stage of the universe. In the simplest analysis we do not take into account effects of the initial mass distribution[3] of black holes, but work with the uniform black holes mass $M$. The time evolution 
of the FRW scale factor $a(t)$ of the universe is governed by the equation

$$
\frac{\dot{a}^{2}}{a^{2}}=\frac{8 \pi G}{3}\left(M(t) n_{B H}(t)+\rho_{R}(t)\right)
$$

where $n_{B H}(t)$ is the number density of the black holes at the epoch $t$, and $\rho_{R}(t)$ is the energy density of radiation. The number density of black holes scales as $a^{-3}$, so that

$$
n_{B H}(t)=\frac{N_{B H}}{a^{3}(t)}
$$

where the total number of black holes formed at time $t_{0}$ is $N_{B H}=n_{B H}\left(t_{0}\right) a^{3}\left(t_{0}\right)$.

The mass of a black hole will change with time due to two factors. First, the process of Hawking radiation causes a loss of mass. Secondly, accretion of the surrounding matter by the black hole leads to an increase in its mass. The rate of change of mass of a single black hole can be written as

$$
\dot{M}=4 \pi R_{B H}^{2}\left(-\sigma T_{B H}^{4}+f \rho_{R}\right)
$$

where $R_{B H}$ is the Schwarzschild radius, $\sigma$ is the Stefan-Boltzmann constant, $T_{B H}$ is the Hawking temperature, and $f$ is an $O(1)$ accretion efficiency factor. Relativistic particles and photons surrounding the black hole dominate the energy energy density of the universe. The thermalysed plasma has density $\rho_{R}$ and a temperature $T$ given by

$$
\rho_{R}=\frac{\pi^{2} g T^{4}}{30}
$$

where $g$ is the number of relativistic particle species.

The energy density of the relativistic matter $\rho_{R}$ will evolve both due to the expansion of the universe, as well as because of the absorption or emission by the black holes. The time-variation of $\rho_{R}$ is thus given by

$$
\frac{d}{d t}\left(\rho_{R}(t) a^{4}(t)\right)=-\dot{M} N_{B H} a(t)
$$

When the right-hand side of Eq.(2.3) vanishes, it yields the usual result $\rho_{R} \propto a^{-4}$. This happens when either no black holes are formed, i.e., $N_{B H}=0$, or when the individual black hole masses do not change with the cosmic time.

In our simplistic analysis let us assume that at time $t_{0}$ a certain fraction $\beta$ of the total energy density of the universe is trapped in $N_{B H}$ number of black holes, all of which have approximately the same mass $M_{0}$. The remaining fraction $(1-\beta)$ of the energy goes into relativitic particles. We assume a small value of $\beta$ corresponding to a radiation dominated era at $t_{0}$. Denoting $\rho_{B H}$ as the sum of the energy densities of all the black holes, one has

$$
\begin{gathered}
\rho\left(t_{0}\right)=\rho_{R}\left(t_{0}\right)+\rho_{B H}\left(t_{0}\right) \\
\rho_{R}\left(t_{0}\right)=(1-\beta) \rho\left(t_{0}\right)=\frac{\pi^{2} g}{30} T^{4} \\
\rho_{B H}\left(t_{0}\right)=\beta \rho\left(t_{0}\right)=M_{0} n_{B H}\left(t_{0}\right)
\end{gathered}
$$


The equations governing the cosmological evolution of the universe beyond the time $t_{0}$ are given by the hubble expansion

$$
\frac{\dot{a}^{2}}{a^{2}}=\frac{8 \pi}{3 M_{4}^{2}}\left(\rho_{R}+\frac{M \beta \rho\left(t_{0}\right)}{M_{0}} \frac{a^{3}\left(t_{0}\right)}{a^{3}}\right)
$$

the evolution of radiation density

$$
\dot{\rho_{R}}=-4 \rho_{R} \frac{\dot{a}}{a}-\frac{\dot{M} \beta \rho\left(t_{0}\right)}{M_{0}} \frac{a^{3}\left(t_{0}\right)}{a^{3}}
$$

and the rate of change of black hole mass Eq.(2.3). The above coupled equations have to be integrated for the specific gravity model under consideration. The modified geometry for the black holes alters the evaporation and accretion rates. Further, the cosmological evolution of the scale factor could also be altered in a model-dependent way, as we would discuss later in context of a braneworld model, and also in context of scalar-tensor models.

\section{Primordial black holes in a braneworld model}

The cosmology of the RS-II model[13] entails a modified high energy phase in the early radiation dominated era of the universe during which the right hand side of the Einstein equation contains terms that are quadratic in the brane energy momentum tensor[14]. Other modifications include the so-called "dark-radiation" term which is given by the projection of the bulk Weyl tensor. Transition to the standard radiation dominated era takes place when $t \geq t_{c} \equiv l / 2$. Such a modified high energy evolution has rich consequences for the physics of the early universe[14]. In particular, the inflationary scenario is altered, allowing the possibility of steep inflaton potentials to accomplish the desired features[15]. Constraints on the duration of the brane dominated high energy phase are enforced by the necessity of conforming to the standard cosmological features at late times.

The effective 4-dimensional Einstein tensor on the brane is given by[14]

$$
G_{\mu v}=\frac{8 \pi}{M_{4}^{2}} \tau_{\mu v}+\kappa^{4} \Pi_{\mu v}-E_{\mu \nu}
$$

where $\tau_{\mu v}$ is the brane energy-momentum tensor; $\Pi_{\mu v}$ is quadratic in the brane EM tensor; and $E_{\mu \nu}$ is the projection of the 5-d Weyl tensor. The 4-dimensional Planck's mass $M_{4}$ is related to the gravitational coupling constant $\kappa$ and the AdS length $l$ by $\frac{8 \pi}{M_{4}^{2}}=\frac{\kappa^{2}}{l}$. For the flat FriedmannRobertson-Walker metric on the brane, the Friedmann equation is given by

$$
H^{2}=\frac{8 \pi}{3 M_{4}^{2}}\left(\rho+\frac{\rho^{2}}{2 \lambda}+\rho_{K K}\right)+\frac{\Lambda_{4}}{3}
$$

with $H$ being the Hubble constant, and $\rho$ the energy density. $\rho_{K K}$ is the effective energy density coming from the bulk Weyl tensor, $\lambda \equiv 3 M_{5}^{6} / 4 \pi M_{4}^{2}$ is the brane tension, and $\Lambda_{4}$ the effective $4-\mathrm{d}$ cosmological constant on the brane. The AdS curvature radius $l$ is given by the bulk cosmological constant $\Lambda_{5}$ and 5-d Planck mass $M_{5}$ as $\Lambda_{5}=-\left(3 M_{5}^{3}\right) /\left(4 \pi l^{2}\right)$. The induced 4-d cosmological constant $\Lambda_{4}$ is given by

$$
\Lambda_{4}=3\left(\frac{M_{5}^{6}}{M_{4}^{4}}-\frac{1}{l^{2}}\right)
$$


Setting $\Lambda_{4}=0$, one obtains a relation between the brane tension and AdS radius given by

$$
\lambda^{-1 / 4}=\left(\frac{4 \pi}{3}\right)^{1 / 4}\left(\frac{l}{l_{4}}\right)^{1 / 2} l_{4}
$$

Nucleosynthesis constrains the "dark energy" term $\rho_{K K}$ to be negligible compared to the radiation density $\rho[16]$. In the following analysis we will neglect $\rho_{K K}$.

Assuming a radiation dominated equation of state, the solutions for the Friedmann equation are given by

$$
\rho_{R}=\frac{3 M_{4}^{2}}{32 \pi t\left(t+t_{c}\right)}
$$

for the energy density, and

$$
a=a_{0}\left[\frac{t\left(t+t_{c}\right)}{t_{0}\left(t_{0}+t_{c}\right)}\right]^{1 / 4}
$$

for the scale factor $a$ during the radiation dominated era, and where $t_{c} \equiv l / 2$ effectively demarcates the brane dominated "high energy" era from the standard radiation dominated era. For times earlier that $t_{c}$, i.e., $t \leq t_{c}$ (or $\rho \geq \lambda$ ), one has the non-standard high energy regime during which the radiation density and the scale factor evolve as

$$
\rho_{R}=\frac{3 M_{4}^{2}}{32 \pi t_{c} t}
$$

and

$$
a=a_{0}\left(\frac{t}{t_{0}}\right)^{1 / 4}
$$

respectively. As a consequence, the time-temperature relation also gets modified during the brane dominated high energy era, i.e., $T \propto t^{-1 / 4}$. But, for times much later than $t_{c}$, i.e., $t>>t_{c}$ (or $\rho<<\lambda$ ), one gets back the standard radiation dominated cosmological evolution given by

$$
\rho_{R}=\frac{3 M_{4}^{2}}{32 \pi t^{2}}
$$

and

$$
a=a_{0}\left(\frac{t}{\left(t_{0} t_{c}\right)^{1 / 2}}\right)^{1 / 2}
$$

Our purpose here is to explore the evolution of primordial black holes in this braneworld scenario.

The formation and evolution of black holes is an interesting and complex issue of investigation in braneworld cosmology. Horizon sized density perturbations in the high energy phase could lead to the formation of black holes on the brane by the process of gravitational collapse. The geometry of such black holes is inherently 5-dimensional[17]. The horizon radii of such black holes is proportional to the square root of their masses, a feature that modifies the Hawking temperature, and consequently slows down the evaporation process[18]. Note however, that the behaviour of large black holes that are super-horizon sized having radius much greater than the Ads curvature radius could be completely different inasmuch as they may evaporate out rapidly as a consequence 
of Ads-CFT correspondence[19]. We will restrict our attention here to black holes which are subhorizon sized at the time of formation. Our analysis here deals with the evolution of black holes once they are formed in the high energy braneworld regime.

Let us consider the evolution of a single primordial black hole which is formed with a subhorizon mass in the high energy radiation dominated era. Such a black hole will have a geometry with an altered mass-radius relarionship given by[18]

$$
r_{B H}=\left(\frac{8}{3 \pi}\right)^{1 / 2}\left(\frac{l}{l_{4}}\right)^{1 / 2}\left(\frac{M}{M_{4}}\right)^{1 / 2} l_{4}
$$

The black hole will accrete the surrounding radiation with a rate proportional to the surface area of the black hole times the energy density of radiation. The Hawking evaporation rate, as for the case of standard black holes, is proportional to the surface area times the fourth power of temperature[20]. The Hawking temperature is given by $T_{B H}=\left(1 / 2 \pi r_{B H}\right)$. Taking into account these effects of accretion and evaporation together, the rate of change of mass $\dot{M}$ of a braneworld black hole is given by

$$
\dot{M}=4 \pi r_{B H}^{2}\left(-g_{\text {brane }} \sigma T_{B H}^{4}+f \rho_{R}\right)
$$

where $g_{\text {brane }}$ is effective number of particles that can be emitted by the black hole (we assume that the black holes can emit massless particles only and take $\left.g_{\text {brane }}=7.25[18]\right), f$ is the accretion efficiency $(0 \leq f \leq 1)[10]$, and $\sigma$ is the Stefan-Boltzmann constant. The black hole aslo evaporates into the bulk, with a rate proportional to $4 \pi r_{B H}^{2} g_{b u l k} T_{B H}^{5}$. However, this term is subdominant even for very small black holes[18], and has negligible effect on their lifetimes.

Substituting the expressions for the black hole radius (Eq.(3.11)), and the energy density of radiation (3.7), the black hole rate equation (3.12) in the radiation dominated high energy braneworld era can be written as[9]

$$
\dot{M}=-\frac{A M_{4}^{2}}{M t_{c}}+\frac{B M}{t}
$$

where $A \simeq\left(3 /(16)^{3} \pi\right)$ and $B \simeq(2 f / \pi)$ are dimensionless numbers. The exact solution for the black hole rate equation was obtained in Ref.[9]. It was shown that a black hole grows in size by the accretion of radiation during the high energy radiation dominated era, with its mass given by $[10,9]$

$$
\frac{M(t)}{M_{0}} \simeq\left(\frac{t}{t_{0}}\right)^{B}
$$

Now let us consider a number density $n_{B H}$ of primordial black holes exchanging energy with the surrounding radiation by accretion and evaporation. The number density of black holes $n_{B H}(t)$ scales as $a(t)^{-3}$, and thus for a radiation dominated evolution on the brane, one gets $\left(n_{B H}(t) / n_{B H}\left(t_{0}\right)\right)=\left(t_{0} / t\right)^{3 / 4}$, since $a(t) \propto t^{1 / 4}$. The net energy in black holes grows since accretion dominates over evaporation. The condition for the universe to remain radiation dominated (i.e., $\left.\rho_{B H}(t)<\rho_{R}(t)\right)$ at any instant $t$ can be derived to be[9]

$$
\beta<\frac{\left(t_{0} / t\right)^{B+1 / 4}}{1+\left(t_{0} / t\right)^{B+1 / 4}}
$$


If the value of $\beta$ exceeds the above bound, there ensues an era of matter (black hole) domination in the high energy braneworld phase. Such a phase of matter domination should definitely be over by the time of nucleosynthesis for the cosmology to be viable.

The growth of the black holes in the radiation dominated era due to accretion slows down with time, since the surrounding radiation density gets diluted. The rate of evaporation is also insignificant for a wide range of $M_{0}$ at this stage since the black hole masses could have grown by several orders of magnitude from their initial values. It is expected that there ensues an era during which the black hole mass stays nearly constant over a period of time, as in case of primordial black holes in standard cosmology[5]. The accretion rate is smaller for the braneworld case since the surface area is $\propto M$ instead of $M^{2}$ for $4 D$ black holes. Moreover, the evaporation rate is $\left(\propto M^{-1}\right)$ instead of $M^{-2}$. Hence, the black hole mass will stay for while near a maximum value $M_{\max }$ reached at time $t_{t}$ before evaporation starts dominating. The expression for the lifetime $t_{\text {end }}$ of a black hole in this scenario is given by[9]

$$
\frac{t_{\text {end }}}{t_{4}} \simeq \frac{4}{A}(2 \sqrt{2})^{B}\left(\frac{M_{0}}{M_{4}}\right)^{2-B} \frac{t_{c}}{t_{4}}\left(\frac{t_{t}^{2}}{t_{c} t_{4}}\right)^{B}
$$

It is important to note that the modified evaporation law also contributes to the increased lifetime for braneworld black holes[18]. However, the effect of accretion is more significant, as can be seen by comparing the lifetime of a 5-d black hole in the presence of accretion to the the lifetime due to purely an altered geometry[9]. Depending upon the values taken by the parameters $t_{c}$ and $l$, one could obtain several interesting examples of primordial black holes surviving till various cosmologically interesting eras $[10,9]$. One particular choice worth mentioning is that of a black hole formed with an initial mass $M_{0}=10^{8} M_{4} \simeq 10^{3} \mathrm{~g}$, that will survive up to the present era if one chooses $\left(l / l_{4}\right) \simeq 10^{30}$.

At any particular era, the surviving black holes would contribute a portion to the the total energy density as dark matter in the universe. If the black holes are produced with an initial mass spectrum, then one would have evaporating black holes at different eras. Hawking radiation from these evaporating black holes would on one hand produce all kinds of particles including heavier ones which could lead to baryogenesis, and on the other, could contribute significantly to the background photons, thus diluting the baryon to photon ratio. Observational constraints impacting different cosmological eras could be used to impose restrictions on the initial mass spectrum of braneworld black holes in a manner similar to the primordial black holes in standard cosmology. Clancy et al[11] have shown how standard constraints are modified in the case of braneworld cosmology. There have been further studies on the impact of braneworld primordial black holes on the high energy diffuse photon background and cosmic ray antiprotons[21]. It has been also shown how the high energy era dynamics is conducive to the formation of black hole binaries[22]. Such binaries during their coalescence may emit gravitational waves amenable for detection[23].

\section{Primordial black holes in a scalar tensor model}

Scalar-tensor models (or Generalyzed Jordan-Brans-Dicke (JBD) models) are obtained in the low energy limit of higher dimensional theories. The JBD[24] theory is one of the earliest and most 
well-motivated alternatives of the Einstein theory of gravitation. The value of the gravitational "constant" is set by the inverse of a time-dependent classical scalar field with a coupling parameter $\omega$. General relativity is recovered in the limit of $\omega \rightarrow \infty$. Solar system observations impose lower bounds on $\omega[25]$. String theoretic[26] and Kaluza-Klein[27] models after compactification of the extra dimensions yield several variants of scalar-tensor models in which the the scalar field coupling $\omega$ may become dynamical, and also models with potential terms for the JBD scalar field.

The feasibility of black hole solutions in JBD theory was first discussed by Hawking[28]. The coexistence of black holes with a long range scalar field in cosmology has interesting consequences [29], and the black holes themselves could be used to constrain the variation of fundamental constants[31]. Another interesting issue of gravitational memory of black holes in JBD theory has also been studied[30]. Since JBD models entail modification of the standard cosmological evolution, it is expected that the evolution of PBHs in JBD models and their associated consequences on various cosmological processes could be substantially modified compared to the case of standard cosmology.

Let us consider a generalysed JBD action given by

$$
S=\frac{1}{16 \pi G} \int d^{4} x \sqrt{-g}\left[\phi R-\frac{\omega(\phi)}{\phi}\left(\partial_{\mu} \phi\right)^{2}\right]+S_{\text {matter }}
$$

where $S_{\text {matter }}$ corresponds to the action of the relativistic fluid of particles $(p=\rho / 3)$ in the flat $(k=0)$ FRW early universe with scale factor $a$. The Friedman equation and the equation of motion for the JBD field $\phi$ obtained from the above action are given respectively by

$$
\frac{\dot{a}^{2}}{a^{2}}+\frac{\dot{a} \dot{\phi}}{a \phi}-\frac{\omega \dot{\phi}^{2}}{6 \phi^{2}}=\frac{\rho}{3 \phi}
$$

and

$$
\ddot{\phi}+3 \frac{\dot{a} \dot{\phi}}{a}=\frac{\rho-3 p}{2 \omega+3}-\frac{\dot{\omega} \dot{\phi}}{2 \omega+3}
$$

with the energy conservation equation given by

$$
\dot{\rho}+3 \frac{\dot{a}}{a}(\rho+p)=0
$$

For a radiation dominated evolution, one has $\rho \approx \rho_{R} \propto a^{-4}$, and $p=\rho / 3$. Assuming power law solutions for the scale factor $a(t)$, the JBD field $\phi(t)$, and also the coupling parameter $\omega(t)$ one can obtain[32] the following time-dependences of these quantities:

$$
\begin{gathered}
a(t)=a_{i}\left(\frac{t}{t_{i}}\right)^{\frac{3}{\omega_{i}+6}} \\
\phi(t)=\phi_{i}\left(\frac{t}{t_{i}}\right)^{-\frac{3}{\omega_{i}+6}} \\
2 \omega(t)+3=\left(2 \omega_{i}+3\right)\left(\frac{t}{t_{i}}\right)^{\frac{2 \omega_{i}+3}{\omega_{i}+6}}
\end{gathered}
$$


where the sbscript $i$ indicates the initial values of the variables. In order to minimize the departure from the standard radiation dominated evolution, we choose $\left|\omega_{i}\right| \ll 1$. Without loss of generality, we set $\phi_{i}=\xi M_{p l}^{2}$, where $M_{p l}=G^{-1 / 2}$ is the Planck mass (corresponding to the present value of $G$ ). Note that the solution for the JBD field $\phi$ indicates the strengthening of gravity with the increase of cosmic time. Such a behaviour for $\phi$ arises not just from the specific model (4.1) used here, but is a rather generic feature of several generalyzed JBD or scalar tensor models with a potential for the scalar field which rolls down the slope leading to the increase of $G$ with time[33].

We now consider the evolution of PBHs in the cosmological background governed by the above solutions (4.5),(4.6) and (4.7). We assume that the PBH density is low enough to ensure radiation domination. The rates of accretion and evaporation by a JBD black hole can be obtained by identifying $r_{B H}=2 M / \phi$ as the black hole radius, and $T=\phi /(8 \pi M)$ as the Hawking temperature. The complete evolution for the PBH is described by[12]

$$
\dot{M}=-\frac{A}{M^{2} t}+\frac{B M^{2}}{t}
$$

It is apparent from Eq.(4.8) that for PBHs with initial mass $M_{i}<(A / B)^{1 / 4}$, the rate of evaporation exceeds that of accretion. Note that though the rate of evaporation decreases with time, it is still higher than the corresponding rate in standard cosmology. This is because the Hawking temperature $T=\phi /(8 \pi M)$ is larger for JBD PBHs for large $\phi$. Hence, a PBH with initial mass $M_{i}<(A / B)^{1 / 4}$ evaporates out much quicker, with a lifetime $t_{\text {evap }}$ given by

$$
\frac{t_{\text {evap }}}{t_{i}}=\exp \left[\frac{1}{3 A}\left(\frac{M_{i}}{M_{p l}}\right)^{3}\left(\frac{t_{p l}}{t_{i}}\right)\right]
$$

However, PBHs with initial mass $M_{i}>(A / B)^{1 / 4}$ experience monotonic growth with accretion dominating over evaporation throughout the period of validity of Eq.(4.8), i.e., throughout the radiation dominated era. The growth law for the PBHs was obtained in Ref.[12], leading to the maximum mass achieved by a PBH of initial mass $M_{i}$, given by

$$
\frac{M_{\max }}{M_{i}} \approx \frac{1}{1-B M_{i} \ln \left(t_{e q} / t_{i}\right)}
$$

In the matter dominated era, $\rho_{M} \sim a^{-3}$ and $p=0$. A set of solutions for the JBD cosmological equations (4.2), (4.3) and (4.4) is given by[32]

$$
\begin{gathered}
a(t)=a\left(t_{e q}\right)\left(\frac{t}{t_{e q}}\right)^{\frac{2}{3}} \\
\phi(t)=\phi\left(t_{e q}\right)\left(\frac{t}{t_{e q}}\right)^{-\frac{4}{3}} \\
\omega(t)=-\omega\left(t_{e q}\right)\left(\frac{t}{t_{e q}}\right)^{\frac{4}{3}}
\end{gathered}
$$

Matching these solutions with those obtained in the radiation dominated era given by Eqs.(4.5),(4.6) and (4.7), one has $\phi\left(t_{e q}\right)=\xi M_{p l}^{2}\left(t_{i} / t_{e q}\right)^{1 / 2}$ and $\omega\left(t_{e q}\right)=\omega_{i}\left(t_{e q} / t_{i}\right)^{1 / 2}$. Substitution of the solution 
for the JBD field $\phi$ leads to the lifetime for the PBHs $t_{\text {evap }}$ given by[12]

$$
t_{\text {evap }}=t_{\text {eq }}\left[1-\frac{5}{3} \frac{M_{i}^{3}}{\left(1-B M_{i} \ln \left(t_{e q} / t_{i}\right)\right)^{3}\left(A t_{i} M_{p l}^{4}\right)}\right]^{-3 / 5}
$$

The rate of evaporation is again faster, as in the radiation dominated era, than the case in standard cosmology (where $t_{\text {evap }} \sim M_{\text {max }}^{3}$ ).

The overall lifetime of a JBD PBH has an interesting comparison to a PBH lifetime in standard cosmology. A JBD PBH with initial mass $M_{i}<(A / B)^{1 / 4}$, evaporates out quicker than a PBH in standard cosmology, being unable to accrete in the radiation dominated era. However, when the initial mass exceeds $(A / B)^{1 / 4}$, accretion proceeds effectively and dominates over evaporation in the radiation dominated era increasing the $\mathrm{PBH}$ mass at the time of matter-radiation equality. In the matter dominated era, though the evaporation rate of JBD PBH is faster, it starts to evaporate out much later in standard cosmology (without accretion). Thus, the lifetime for a $\mathrm{PBH}$ with $M_{i}>$ $(A / B)^{1 / 4}$ is enhanced in the JBD scenario compared to the case in standard cosmology. Certain interesting examples of evolving PBHs in JBD cosmology were observed in Ref.[12]. It was shown that for a PBH with $M_{i}<(A / B)^{1 / 4}$ to survive the radiation dominated era, one requires $M_{i} \geq$ $10^{13} M_{p l}$. A PBH which forms at time $t_{E W}$ with an initial mass of the order of the cosmological horizon mass at $t_{E W}$ satisfies the condition $M_{i}>(A / B)^{1 / 4}$, and accretes radition. Though the actual growth of mass turns out to be negligible for such a $\mathrm{PBH}$, accretion does play an important role enabling it to survive for much longer $\left(t_{\text {evap }}>t_{\text {now }}\right)$, with the PBH thus being a component of cold dark matter.

\section{Conclusions}

The subject of primordial black holes is a fascinating one that has received the attention of a number of researchers over the last few decades. In this article we have provided a description of the braneworld cosmological evolution with primordial black holes. The modified mass-radius relationship for the induced 4-dimensional Myers-Perry black holes leads to slower evaporation and longer lifetimes compared to standard Schwarzschild black holes[18]. The accretion of radiation in the high energy phase could be effective because the growth of black hole mass is smaller than the growth of the mass in the Hubble volume in braneworld evolution[9, 10]. Thus, a large fraction of primordial black holes may survive up to much later eras. The decaying black holes affect several cosmological processes at different eras, and hence the observational abundance of different species, for example, the background high enery photons[21], could be used to put constraints on the initial mass spectrum of the black holes. These contraints have to be evaluated considering the altered cosmological evolution in the braneworld scenario, and therefore could be significantly modified compared to similar constraints in standard cosmology[11]. The exchange of energy of the black holes with the surrounding radiation in the high energy era leads to the formation of binaries[22]. Such binaries have masses in the sub-lunar range, and gravitational waves emitted during their coalescence come in the detectable range[23].

We have also discussed the evolution of PBHs in scalar-tensor cosmology and obtained a cut-off value for the initial mass which decides whether the PBHs could survive today. An interesting consequence of our analysis is that no $\mathrm{PBHs}$ evaporate during the matter dominated era, and 
hence there is no impact on the cosmic microwave background radiation due to evaporating PBHs. Though our results are in the context of a particular scalar-tensor model, the sort of evolution obtained for the scalar field $\phi$ is generic to other models as well, in particular those following from higher dimensional theories[33]. With the possibility of future observations of actually finding or ruling out scalar-tensor models by determining $\omega$, it would indeed be exciting to re-work the standard observational constraints[2] on the density of PBHs at several cosmological eras in the this scenario which could be viable model in the low energy limit of string theory.

\section{References}

[1] B. J. Carr, Lect. Notes Phys. 631, 301 (2003).

[2] B. J. Carr and J. E. Lidsey, Phys. Rev. D48, 543 (1993); A. M. Green and A. R. Liddle, Phys. Rev. D56, 6166 (1997).

[3] K. Jedamzik and J. C. Niemeyer, Phys. Rev. D59 124014 (1999); A. M. Green and A. R. Liddle, Phys. Rev. D60, 063509 (1999).

[4] Ya. B. Zeldovich and I. D. Novikov, Astron. Zh. 43, 758 (1966); S. W. Hawking, Mon. Not. Roy. Ast. Soc. 152, 75 (1971); B. J. Carr and S. W. Hawking, Mon. Not. Roy. Ast. Soc. 168, 399 (1974); S. W. Hawking, I. G. Moss and J. M. Stewart, Phys. Rev. D26, 2681 (1982); H. Kodama, M. Sasaki and K. Sato, Prog. Theor. Phys. 68, 1979 (1982).

[5] A. S. Majumdar, P. Das Gupta and R. P. Saxena, Int. J. Mod. Phys. D4, 517 (1995); P. S. Custodio and J. E. Horvath, Phys. Rev. D58, 023504 (1998); Phys. Rev. D60, 083002 (1999); N. Upadhyay, P. Das Gupta and R. P. Saxena, Phys. Rev. D60, 063513 (1999).

[6] D. Blais, C. Keifer and D. Polarski, Phys. Lett. B535, 11 (2002).

[7] N. Afshordi, P. McDonald, and D. N. Spergel, Astrophys. J. 594, L71 (2003).

[8] R. Bean and J. Magueijo, Phys. Rev. D66, 063505 (2002).

[9] A. S. Majumdar, Phys. Rev. Lett 90, 031303 (2003).

[10] D. Clancy, R. Guedens and A. R. Liddle, Phys. Rev. D66, 083509 (2002).

[11] D. Clancy, R. Guedens and A. R. Liddle, Phys. Rev. D68, 023507 (2003).

[12] A. S. Majumdar, D. Gangopadhyay and L. P. Singh, Mon. Not. Roy. Astron. Soc. 385, 1467 (2008).

[13] L. Randall and R. Sundrum, Phys. Rev. Lett. 83, 4690 (1999).

[14] R. Maartens, [gr-qc/0312059] Living Rev. Rel. 7, 1 (2004).

[15] E. J. Copeland, A. R. Liddle and J. E. Lidsey, Phys. Rev. D64 023509 (2001); A. S. Majumdar, Phys. Rev. D64, 083503 (2001); V. Sahni, M. Sami and T. Souradeep, Phys. Rev. D65, 023518 (2002); J. E. Lidsey, [astro-ph/0305528] Lect. Notes Phys. 646, 357 (2004).

[16] P. Binetruy, C. Deffayet, U. Ellwanger and D. Langlois, Phys. Lett. B477, 285 (2000); J. D. Barrow and R. Maartens, Phys. Lett. B532, 153 (2002).

[17] R. C. Myers and M. J. Perry, Ann. Phys. 172, 304 (1986).

[18] R. Guedens, D. Clancy and A. R. Liddle, Phys. Rev. D66, 043513 (2002).

[19] T. Tanaka, Prog. Theor. Phys. Suppl. 148, 307 (2003); R. Emparan, A. Fabbri and N. Kaloper, JHEP 0208, 043 (2002); R. Emparan, J. Garcia-Bellido and N. Kaloper, JHEP 0301, 079 (2003). 
[20] For a review of braneworld black hole evaporation, see, P. Kanti, Int. J. Mod. Phys. A19, 4899 (2004).

[21] Y. Sendouda, S. Nagataki and K. Sato, Phys. Rev D68, 103510 (2003); Y. Sendouda, K. Kohri, S. Nagataki and K. Sato, Phys. Rev. D71, 063512 (2005).

[22] A. S. Majumdar, A. Mehta and J-M. Luck, Phys. Lett. B 607, 219 (2005).

[23] K. T. Inoue and T. Tanaka, Phys. Rev. Lett. 91, 021101 (2003).

[24] C. H. Brans and R. H. Dicke, Phys. Rev. 124, 925 (1961).

[25] C. M. Will, Living. Rev. Relativity 9, 3 (2006).

[26] See, for example, M. Gasperini and G. Veneziano, in Beyond the Big-bang, ed. R. Vaas (Springer-Verlag, Heidelberg, 2007).

[27] Modern Kaluza-Klein Theories, eds. T. Appelquist, A. Chodos and P. G. O. Freund (Addison-Wesley, 1987).

[28] S. W. Hawking, Comm. Math. Phys. 25, 167 (1972).

[29] K. G. Zloshchastiev, Phys. Rev. Lett. 94, 121101 (2005).

[30] J. D. Barrow and B. J. Carr, Phys. Rev. D 54, 3920 (1996); T. Harada, C. Goymer, and B. J. Carr, Phys. Rev. D 66, 104023 (2002).

[31] J. H. MacGibbon, Phys. Rev. Lett. 99, 061301 (2007).

[32] B. K. Sahoo and L. P. Singh, Mod. Phys. Lett. A17, 2409 (2002); Mod. Phys. Lett. A18, 2725 (2003).

[33] A. S. Majumdar and S. K. Sethi, Phys. Rev. D46, 5315 (1992); A. S. Majumdar, T. R. Seshadri and S. K. Sethi, Phys. Lett. B312, 67 (1993); A. S. Majumdar, Phys. Rev. D 55, 6092 (1997). 